\title{
Comment on: "Cost Effectiveness of Tiotropium in Patients with Asthma Poorly Controlled on Inhaled Glucocorticosteroids and Long-Acting $\beta$-Agonists"
}

\author{
Carl Samuelsen $^{1} \cdot$ Eric D. Bateman ${ }^{2} \cdot$ Ian Pavord $^{3} \cdot$ Adam Lloyd $^{4}$ • \\ Mike Baldwin ${ }^{5}$ Dirk Esser ${ }^{5}$
}

Published online: 30 December 2015

(C) The Author(s) 2015. This article is published with open access at Springerlink.com

Dear Editor

Re: Willson et al. [1]

\section{Introduction}

When performing a re-analysis of our study data, we found an error in the calculation of the transition matrices in our published analyses relating to how patients' asthma-control states at weekly study visits had been imputed and subsequently used in our original model. We have therefore reanalysed the data using revised transition matrices and also performed additional sensitivity analyses to confirm the reliability and validity of the conclusions.

\section{Re-Analysis of Study Data}

The original Bayesian biphasic model was based on the observed number of weekly patient transitions in the clinical trials using six-question Asthma Control Questionnaire (ACQ-6) results and suggested that, in both treatment arms,

Dirk Esser

dirk.esser@boehringer-ingelheim.com

Boehringer Ingelheim, Asker, Norway

2 Division of Pulmonology, Department of Medicine, University of Cape Town, Cape Town, South Africa

3 Respiratory Medicine, University of Oxford, Oxford, UK

4 Health Economics and Outcomes Research, Real-World Evidence Solutions, IMS Health, London, UK

5 Boehringer Ingelheim, Ingelheim, Germany there was a rapid improvement in the number of patients who were considered to have both controlled and partly controlled asthma in weeks $1-8$, followed by a slower rate of change in weeks 9-48. The re-analysis of study data using the revised transition matrices predicted that tiotropium add-on therapy reduced exacerbations and improved asthma control with an incremental cost-effectiveness ratio of $£ 28,383$ (obtained at 2012 prices) per quality-adjusted life-year (QALY) gained, which is within the commonly accepted $£ 20,000-£ 30,000$ per QALY gained willingness-to-pay threshold used in the UK [2]. There was a $52 \%$ likelihood of cost-effectiveness at a willingness-to-pay of $£ 30,000$ per QALY gained and a $31 \%$ likelihood of cost-effectiveness at a willingness-to-pay of $£ 20,000$ per QALY gained, when compared with usual-care treatment.

Although this re-analysis resulted in a higher overall incremental cost-effectiveness ratio, tiotropium was still found to be cost-effective when added to usual care in patients whose asthma remained uncontrolled despite treatment with high-dose inhaled glucocorticosteroids (ICSs) with long-acting $\beta 2$-agonists (LABAs) (budesonide $800 \mu \mathrm{g} /$ formoterol fumarate $24 \mu \mathrm{g}$ or fluticasone propionate $500 \mu \mathrm{g} / \mathrm{salmeterol} 100 \mu \mathrm{g}$ ), in line with the overall conclusions from our original publication. However, this result was associated with greater uncertainty, reducing the likelihood of cost-effectiveness at a willingness-to-pay of $£ 30,000$ per QALY gained from $66 \%$ to $52 \%$.

\section{Additional Sensitivity Analyses}

To address the increased uncertainty, we performed additional sensitivity analyses to explore the robustness of the revised model. 
Table 1 Incremental cost-effectiveness ratios calculated during the re-analysis of study data and additional sensitivity analyses (all values in $£$ per QALY gained)

\begin{tabular}{lll}
\hline Analysis modelling approach & \multicolumn{2}{l}{ ACQ version } \\
\cline { 2 - 3 } & ACQ-6 & ACQ-7 \\
\hline Bayesian monophasic & 20,260 & 17,987 \\
Bayesian biphasic & $28,383^{\mathrm{a}}$ & 24,844 \\
Hybrid & 26,386 & 21,756 \\
Tunnel & 24,685 & 21,759
\end{tabular}

Obtained at 2012 prices

${ }^{\text {a }}$ Corresponds to the re-analysis of study data performed using the methodology outlined in the original publication

The original model used a biphasic approach with different transition matrices for weeks $1-8$ and 9-48. The results of the re-analysis of study data suggest that this approach may have underestimated the early treatment effect and overestimated the late treatment effect. We have therefore performed additional sensitivity analyses using a number of modelling approaches including a Bayesian monophasic model, a hybrid model which combines a biphasic approach for the asthma-control states and a monophasic approach for exacerbations, and a model using tunnelling states. We also examined whether the ACQ version [ACQ-6 or seven-question ACQ (ACQ-7)] affected the results (Table 1).

The results of these additional sensitivity analyses show that tiotropium may be cost-effective when added to usual care in patients whose asthma remains uncontrolled despite treatment with high-dose ICS plus LABA, irrespective of the modelling method used, and so provides additional confidence that the conclusions presented in our published manuscript are valid.
Acknowledgments IMS Health, funded by Boehringer Ingelheim, conducted the original analyses; Adam Lloyd was the guarantor of the overall content. Carl Samuelsen, Mike Baldwin and Dirk Esser conducted the new analyses, as outlined in this letter. Professor Eric Bateman and Professor Ian Pavord provided expertise on the clinical aspects of this study. Carl Samuelsen, Mike Baldwin and Dirk Esser are full-time employees of Boehringer Ingelheim. Adam Lloyd is a full-time employee of IMS Health; Jenny Willson and Tania Krivasi were authors on the original publication but are no longer employees of IMS Health. Professor Eric Bateman is Emeritus Professor of Medicine at the University of Cape Town and received consulting fees or honoraria from Boehringer Ingelheim for the meetings connected with this study. Professor Ian Pavord is Professor of Respiratory Medicine at the University of Oxford and received consulting fees or honoraria from Boehringer Ingelheim for the meetings connected with this study. Medical writing assistance with this letter was supported financially by Boehringer Ingelheim and provided by Lianne Young, BSc (Hons), at Complete HealthVizion under the authors' conceptual direction and based on feedback from the authors.

Open Access This article is distributed under the terms of the Creative Commons Attribution-NonCommercial 4.0 International License (http://creativecommons.org/licenses/by-nc/4.0/), which permits any noncommercial use, distribution, and reproduction in any medium, provided you give appropriate credit to the original author(s) and the source, provide a link to the Creative Commons license, and indicate if changes were made.

\section{References}

1. Willson J, Bateman ED, Pavord I, Lloyd A, Krivasi T, Esser D. Cost effectiveness of tiotropium in patients with asthma poorly controlled on inhaled glucocorticosteroids and long-acting $\beta$ agonists. Appl Health Econ Health Policy. 2014;12(4):447-59.

2. National Institute for Health and Care Excellence. Process and methods guides. The guidelines manual. 2012. http://www.nice.org. uk/article/pmg6/resources/non-guidance-the-guidelines-manual-pdf. Accessed Oct 2015 